\title{
Reaction Selectivity in Heterogeneous Catalysis
}

\author{
Gabor A. Somorjai* and Christopher J. Kliewer \\ Department of Chemistry, University of California, Berkeley, California 94720, and Materials \\ Sciences Division, Lawrence Berkeley National Laboratory, Berkeley, California 94720 \\ E-mail: somorjai@socrates.berkeley.edu \\ TITLE RUNNING HEAD: Selectivity in Heterogeneous Catalysis \\ CORRESPONDING AUTHOR FOOTNOTE: Gabor A. Somorjai, Tel: 510-642-4053. Fax: 510- \\ 643-9668. E-mail: somorjai@ socrates.berkeley.edu.
}

Review for Reaction Kinetics and Catalysis Letters 


\begin{abstract}
.
The understanding of selectivity in heterogeneous catalysis is of paramount importance to our society today. In this review we outline the current state of the art in research on selectivity in heterogeneous catalysis. Current in-situ surface science techniques have revealed several important features of catalytic selectivity. Sum frequency generation vibrational spectroscopy has shown us the importance of understanding the reaction intermediates and mechanism of a heterogeneous reaction, and can readily yield information as to the effect of temperature, pressure, catalyst geometry, surface promoters, and catalyst composition on the reaction mechanism. DFT calculations are quickly approaching the ability to assist in the interpretation of observed surface spectra, thereby making surface spectroscopy an even more powerful tool. HP-STM has revealed three vitally important parameters in heterogeneous selectivity: adsorbate mobility, catalyst mobility, and selective site-blocking. The development of size controlled nanoparticles from 0.8 to $10 \mathrm{~nm}$, of controlled shape, and of controlled bimetallic composition has revealed several important variables for catalytic selectivity. Lastly, DFT calculations may be paving the way to guiding the composition choice for multi-metallic heterogeneous catalysis for the intelligent design of catalysts incorporating the many factors of selectivity we have learned.
\end{abstract}

\title{
1. Introduction
}

Ferenc Màrta was responsible for my first visit to Hungary in 1987 after my 31 year absence. His kind invitation to attend a photochemistry conference renewed my friendship and interaction with many in this chemistry community which has continued uninterrupted since that time. Under the leadership of Professor Márta and his successor Gábor Pálinkás the 
Chemistry Institute of the Hungarian Academy of Sciences gained international reputation as one of the outstanding research centers in Europe. Ferenc Márta has lit the flame of the renaissance of chemistry that permitted Hungary to be counted among the leaders of research in chemistry.

As we continue into the twenty-first century, heterogeneous catalysis has never held a more pivotal role. Not only do a great many industrial processes rely on heterogeneous catalysts today, but as the global concerns of energy production and conversion, alternative energy sources, and climate change garner attention, the importance of heterogeneous catalysis is again paramount. Although many reactions can be promoted with either homogeneous or heterogeneous catalysis, the latter generally allows for less waste, fewer toxic reagents, and easier retrieval and recycling of the catalyst ${ }^{1}$. This allows for a more "green" chemistry. In this same regard, the ultimate goal in heterogeneous catalysis must be for $100 \%$ selectivity for the desired product in a multi-pathway reaction, eliminating unwanted byproducts. A fundamental understanding of selectivity in heterogeneous catalysis also plays a large role in emerging energy technologies as well. For instance, the efficiency of compact mixed-reactant fuel cells vitally depends on the selectivity with which the anode and cathode electrocatalysts can carry out fuel oxidation and oxidant reduction within the same cell ${ }^{2}$.

The understanding of how a heterogeneous catalyst works at the molecular level grew exponentially during the twentieth century thanks in large part to the development of ultrahigh vacuum (UHV) surface science techniques. Advancements in high vacuum technology resulted in the ability to keep a catalyst surface clean long enough to do interrogative experiments. This resulted in techniques obtaining surface-specific information by using electron, photon, and ion scattering. For instance, adsorbing one or more reactants to a clean 
metal surface, which was then probed with high resolution electron energy loss spectroscopy (HREELS), resulted in the vibrational signature of surface-bound molecules and intermediates ${ }^{3}$ (Fig 1).

The limitation of the electron and ion scattering techniques, however, was that they were bound to the low pressure regime due to the large collisional cross-section of these probes with the gas or liquid phase molecules. This created a "gap" in pressure between the fundamental research being done in surface science laboratories and the pressures at which catalytic reactions would be carried out industrially. Being limited to the low-pressure regime made it difficult to study such reactions as hydrocarbon reforming which have very low reaction probabilities ${ }^{4}$ necessitating high pressure conditions. Further, short lived surface reaction intermediates can only be observed under conditions of low temperature or high pressure, but low temperature conditions can yield reduced adsorbate mobility, reducing the ability of the adsorbates to follow the reaction mechanism. In addition to the mobility of the adsorbate, the mobility of the substrate metal atoms is also of concern. Thus a large portion of the reaction phase-space was unavailable until the more recent advent of in-situ surface science techniques which have allowed the probing of surfaces and surface-bound molecules even at the higher pressure ranges encountered during real industrial processes.

It is the goal of this review to demonstrate how the current state of development with in-situ surface science techniques in combination with recent advances and accomplishments in density functional theory (DFT) calculations are paving the way to a new era in heterogeneous catalysis in which the full mechanistic understanding of chemical reactions occurring over heterogeneous catalysts will allow for the logical design of optimized catalysts, with the goal being a catalyst which is $100 \%$ selective for the desired product while maintaining high activity 
and stability. It is not our purpose to provide a complete and historic review of the development of surface science, but to assess the current state of the field.

Selectivity is defined as the rate of a reaction along a particular pathway divided by the sum of the rates along all reaction pathways. Of the three attributes which make a good catalyst, activity, selectivity, and stability, it is selectivity which is the least well understood as of yet in heterogeneous catalysis. Most of the insights of twentieth century research were aimed at increasing catalyst activity to boost production. But the demand for selective heterogeneous catalysis has become more apparent in many applications.

It is therefore of paramount importance that a molecular-level understanding of the factors which control the selectivity of a reaction be reached to move the science of selective heterogeneous catalysis out of the phenomenological or "trial and error" mode and into a more intelligent design of catalysts.

\section{Experimental Advancements and Understanding Selectivity in Heterogeneous}

\section{Catalysis}

Several new advancements in experimental and theoretical techniques have been developed and are now being used which have greatly enhanced our ability to study heterogeneous catalysis on a molecular level. These techniques are bringing about a new understanding of the factors which control heterogeneous selectivity. High pressure surface spectroscopy such as sumfrequency generation vibrational spectroscopy (SFG-VS), high pressure scanning tunneling microscopy (HP-STM), advancements in the controlled synthesis of monodispersed nanoparticles with controlled size, shape, and composition, and advancements in the use of DFT 
calculations to guide experiments will be discussed. It is the merging of all these techniques which will pave the way to full understanding of catalytic selectivity.

\section{Sum-Frequency Generation Vibrational Spectroscopy.}

Sum-frequency generation vibrational spectroscopy (SFG-VS) is a nonlinear spectroscopy technique in which two high-energy pulsed laser beams are overlapped in spacially and temporally on an interface of interest ${ }^{5}$. Due to the properties of the nonlinear susceptibility tensor, media with inversion symmetry such as isotropic gases or bulk metal crystals cannot generate a SFG-VS signal. Thus, the entire signal is generated at the interface. The infraredvisible SFG-VS process (Fig. 2) can be thought of as an infrared excitation followed by an antistokes Raman relaxation process, the result of which is emitted radiation at the sum of the two incoming frequencies.

The technique of infrared-visible SFG-VS has been applied in our laboratory to many catalytic systems at high pressure: the oxidation of $\mathrm{CO}$ on Pt single crystals ${ }^{6-8}$, the hydrogenation of ethylene over Pt single crystals ${ }^{9,10}$, the hydrogenation and dehydrogenation of isobutene ${ }^{11}$ over $\operatorname{Pt}(111)$, the hydrogenation and dehydrogenation of cyclohexene ${ }^{12,13}$ over $\operatorname{Pt}(111)$ and $\operatorname{Pt}(100)$, the hydrogenation of benzene to cyclohexene and cyclohexane ${ }^{14,15}$ over $\operatorname{Pt}(111)$ and $\operatorname{Pt}(100)$, and the hydrogenation of pyrrole to pyrrolidine and butylamine ${ }^{16}$ over Pt and Rh single crystals. As can be seen, the initial reaction studies of $\mathrm{CO}$ oxidation and ethylene hydrogenation were oneproduct reactions. But the progressive trend has been to go to more complicated reaction studies in which a molecular understanding can improve our fundamental knowledge about catalytic selectivity. The focus of all of these studies has been to attempt to elucidate the reaction mechanism including the surface reaction intermediates. The goal is to assess how the surface 
intermediates and reaction mechanism change with changing experimental parameters such as catalyst composition, crystal face, pressure, temperature, and the presence of coadsorbates.

As an example of the power of SFG-VS, Figure 3 displays the spectrum of pyrrole during hydrogenation conditions over $\operatorname{Pt}(111)^{16}$. The surface-bound reaction intermediate pyrroline was seen, which was not detected in the gas phase. Evidence of a significant concentration of the product molecules, pyrrolidine and butylamine, was seen to build up on the surface under reacting conditions. This indicates that the desorption of the products is a rate-limiting step, and efforts to reduce the binding energy of the products with the surface should cause an overall increase in reaction rate. The reduction of residence time of the saturated ring product, pyrrolidine, on the surface decreases the probability for forming the ring-cracking product butylamine, improving the overall selectivity for the saturated ring product. These considerations are reminiscent of the drastic rate enhancement of ammonia synthesis over Fe catalysts that was seen upon the addition of potassium as a promoter ${ }^{17}$.

Another method of surface vibrational spectroscopy that currently shows great promise for elucidating surface intermediates under high-pressure conditions is polarization-modulation infrared reflection absorption spectroscopy (PM-IRAS) ${ }^{18-20}$. The two spectroscopic techniques of SFG-VS and PM-IRAS are complimentary. SFG has the advantage that the signal is inherently generated at the surface. However, as the SFG signal depends linearly on the intensity of the incoming infrared radiation, in the presence of high gas pressures and long path-lengths the resulting SFG-VS spectra must be corrected for the attenuation of infrared radiation from the gas phase prior to striking the catalyst surface. PM-IRAS has the advantage of an easily accessible wide spectral range allowing for the probing of amide, $\mathrm{C}=\mathrm{O}, \mathrm{C}=\mathrm{C}, \mathrm{C}-\mathrm{H}$, and $\mathrm{O}-\mathrm{H}$ 
modes simultaneously without changing non-linear crystals in an optical parametric generation/amplification stage as in the case of the SFG-VS apparatus ${ }^{21}$.

\section{DFT Calculated Vibrational Frequencies}

One of the difficulties that arises in more complex heterogeneous multi-pathway reactions with regards to surface-specific spectroscopy is the interpretation of vibrational spectra. Upon adsorption to a metal surface, even molecules which undergo very little chemical change can have vast differences in vibrational signature. Electron donation from the $\mathrm{C}$ - $\mathrm{H} \sigma$-bonds in methyl groups upon molecular adsorption to the platinum surface can induce as much as a $40 \mathrm{~cm}^{-}$ ${ }^{1}$ red-shift in the frequency of the methyl asymmetric stretch as compared with the unperturbed molecule. Zaera et al. have frequently employed the method of halogenating a hydrocarbon in a pre-selected position so that upon dissociative adsorption an alkyl bonded structure is left on the surface $^{22-24}(\text { Fig. } 4)^{25}$. Taking the vibrational spectra of intermediates in this way can provide a reference state with which to judge the spectra taken under catalytic conditions. However, not all possible stable surface intermediates can be prepared in this way, and such commonly suggested reaction intermediates as allyl-type structures are difficult to unambiguously prepare on a surface for a reference spectrum.

As technology becomes faster and more affordable, however, theoretical calculations become more powerful. The accurate calculation of adsorption energies of possible reaction intermediates could guide temperature programmed desorption experiments, aiding in the identification of surface intermediates using their binding energy. This is of course a lowpressure experimental method. DFT calculations can also aid in the interpretation of surface specific spectra by calculating the expected vibrational frequencies of possible surface-bound 
reaction intermediates. There is a long way to go in this field. For instance, the incorporation of vibrational anharmonicities into the calculated vibrational frequencies would greatly aid in the certainty of calculated values, especially in the $\mathrm{C}-\mathrm{H}$ region of the infrared spectrum. However, great strides towards the identification of surface intermediates using theoretical calculations have been taken. For instance, figure 5 demonstrates a study by Loffreda $\mathrm{et}^{\mathrm{al}^{26}}$ in which they ascertained the possible adsorption modes of acrolein to a $\mathrm{Pt}(111)$ surface, and from the energyminimized adsorption structures calculated vibrational frequencies and simulated EELS spectra for comparison to HREELS data. Such calculations can serve to compliment experimental data and make the application of surface-specific spectroscopies even more powerful, extending their use to even more complicated catalytic systems.

\section{High-Pressure Scanning Tunneling Microscopy}

HP-STM has proven to be an invaluable tool to image catalyst surfaces with atomic resolution even under high reactant pressures ${ }^{27-35}$. Figure 6 demonstrates one of the key components of catalytic activity discovered in our laboratory during the selective reaction of

cyclohexene hydrogenation and dehydrogenation over a $\operatorname{Pt}(111)$ single crystal $^{36}$. The crystal was first introduced to $20 \mathrm{mTorr}$ hydrogen and $20 \mathrm{mTorr}$ cyclohexene at room temperature. Under these conditions the surface has been shown by SFG-VS to be dominated by the partially dehydrogenated $\pi$-allyl $\mathrm{C}_{6} \mathrm{H}_{9}$ surface intermediate. The surface is not catalytically active under these reactant pressures, and surface adsorbate ordering is observed in the STM image (panel A). However, in panel B of figure 6, the backpressure of hydrogen has been raised to $200 \mathrm{mTorr}$. Under these conditions, the crystal is catalytically active producing both benzene and cyclohexane. The STM image, taken at a scan speed of $\sim 100 \AA$ A per ms, shows no long range order. The adsorbates are diffusing more quickly than the tip can scan. In panel C, $5 \mathrm{mTorr}$ of 
$\mathrm{CO}$ was added to the reaction mixture which poisons the surface and ends catalytic activity, and once again, a static structure was seen with STM. From this we learn that adsorbate mobility is a key feature of catalytic activity. This, of course, has implications when one considers going to very low temperatures to observe a surface reaction intermediate using low pressure probes typical of UHV surface science. At these temperatures, surface adsorbate mobility will be greatly reduced, and experimental attempts to probe catalytic selectivity may not in some cases extrapolate well to the higher temperature and pressure regime used during industrial catalysis, especially in the study of more complex multi-pathway catalytic reactions.

The second important factor to catalytic selectivity, which is uniquely explored by HP-STM is adsorbate induced catalyst restructuring. The delicate interplay between the generally exothermic process of chemisorption and the endothermic process of stretching and breaking metal bonds will often create a state upon adsorption in which metal atoms are dislocated from their bulk-terminated positions and can lead to major surface restructuring ${ }^{37}$.

The third factor vitally important to catalytic selectivity explored by STM is selective catalyst site-blocking. It has long been known that step sites and kink sites are far more active in bond scission than are terrace sites ${ }^{38-40}$. Figure 7 displays STM images from the work of Vang et $\mathrm{al}^{41}$. Upon exposing $\mathrm{Ni}(111)$ to ethylene, a "brim" forms around the step edge seen in the image resulting from the dissociation of ethylene. However, the authors show that pretreating the surface with $\mathrm{Ag}$ results in the blocking of these sites as can be seen in the figure. Upon ethylene exposure, the Ag treated $\mathrm{Ni}(111)$ catalyst did not create a "brim" of dissociated ethylene. In a multi-pathway reaction where selectivity is paramount, the major effect that steps and kinks play can be investigated by using HP-STM. 


\section{Bridging the Materials Gap: Creation of Nanoparticles of Controlled Size, Shape,}

\section{Composition, and Support.}

Bridging of the "pressure gap" has been discussed in much detail, but an equally important aspect of understanding selectivity as it applies to industrial catalysts is the "materials gap". Industrial heterogeneous catalysts are nanoparticles in the 1-10 nm size range generally loaded onto oxide or carbon supports. This introduces new variable parameters which can affect the catalytic selectivity and thus a molecular level understanding of these factors and their effect on selectivity is important to the field. To this end, our laboratory has refined several synthetic techniques to create size, shape, and composition controlled Pt, Rh, and Pd nanoparticles. Scheme 1 shows schematically various sizes of Pt metal nanoparticles created in our laboratory. We will not go into great detail here on the synthesis of such particles as it is covered in the literature $^{42-45}$. Briefly nanoparticles in the range of $0.8 \mathrm{~nm}$ to $2 \mathrm{~nm}$ have been synthesized using fourth generation hydroxyl terminated polyamidoamine (PAMAM) dendrimers as the capping and templating agent ${ }^{43}$. Nanoparticles in the range of $3 \mathrm{~nm}$ to $8 \mathrm{~nm}$ can be created via colloidal chemistry using the appropriate metal precursor to generate metal ions in solution, and then reducing the ions in the presence of polyvinylpyrolidone (PVP) used as a capping agent ${ }^{45}$ to keep the particles from aggregating. The effect of particle size on the organic selectivity of heterogeneous catalysis has been shown to be quite significant. For instance, figure 8 shows the size dependence of the reaction selectivity in the hydrogenation of pyrrole over monodisperse $\mathrm{Pt}$ and $\mathrm{Rh}$ nanoparticles.

Shape controlled nanoparticles have been created using optimized reduction conditions: cubes, which maximize the abundance of (100) facets, and cuboctahedra, which contain both (111) and (100) facets ${ }^{46}$ have been synthesized and characterized. 
The resulting nanoparticles can be deposited using the Langmuir-Blodgett technique onto a twodimensional support for catalytic studies, or they can be placed into a three-dimensional oxide support such as SBA-15 for high surface area catalytic studies. We are still in the early stages of applying the in-situ surface science techniques described above to these new model catalysts.

One obstacle to the use of SFG-VS or HP-STM in probing the model nanoparticle catalysts was the presence of the hydrocarbon capping layer used to keep the nanoparticles from aggregating. This problem has recently been overcome by the use of a UV/ozone treatment ${ }^{47}$. Figure 9 displays SFG-VS spectra obtained during the hydrogenation of pyridine over TTAB-stabilized cubic platinum nanoparticles $^{48}$. The spectra demonstrate the presence of the pyridinium cation as a surface intermediate during this reaction.

\section{DFT Guided Nanoparticle Composition}

Several new bimetallic structured nanoparticles have been synthesized in our lab. $\mathrm{Rh} / \mathrm{Pd}^{49}$, $\mathrm{Pt} / \mathrm{Pd}^{49}, \mathrm{Ni} / \mathrm{Cu}^{50}$, and $\mathrm{Rh} / \mathrm{Pt}^{51}$ bimetallic nanoparticles have all been synthesized and used in catalytic studies. While fundamental studies using bimetallic catalysts are worthy of attention, recent developments in the research group of Jens Nørskov suggest that theoretical DFT calculations may be able to guide experimental studies by suggesting catalyst combinations which may generate the optimum balance between activity and selectivity for a particular reaction. In a recent article ${ }^{52}$, they were able to apply the technique in attempting to find a better and more cost efficient catalyst for the selective reduction of acetylene in a stream of ethylene as is often encountered in polymer synthesis. The technique hinges on being able to find a suitable parameter (such as heat of adsorption) and scaling relation to simplify the calculations enough to compare various catalyst combinations. In the article mentioned, it was determined that the 
adsorption energy of the methyl species scaled with both the heats of adsorption of ethylene and acetylene so it was used as the simplified parameter to compare catalyst combinations.

This is a promising future direction in heterogeneous catalysis research where theoretical insights could help guide experimentalists through nearly limitless combinations towards those multicomponent catalysts with the desired activity.

\section{A Look to the Future}

We are now in a new era for heterogeneous catalysis research. Surface-specific spectroscopies can be employed in-situ from UHV pressures to above ambient pressures, providing a vibrational spectrum of surface species before, during, and after a catalytic reaction. We can explore the effects of pressure, temperature, catalyst geometry, and coadsorbate promoters on surface intermediates. HP-STM can be employed from UHV to high pressures to gain atomically resolved images of working catalyst surfaces. From the HP-STM three major factors of selectivity in heterogeneous catalysis have emerged, adsorbate mobility, catalyst mobility, and selective site-blocking. The development of controlled nanoparticle synthesis has given us the ability to begin to understand the effects of particle size, catalyst geometry, and bimetallic composition on catalyst selectivity using model catalysts which very closely resemble those used industrially. As DFT theoretical calculations become more complete, they will serve to guide in the interpretation of surface vibrational results, thereby making SFG-VS and PM-IRAS more powerful in identifying surface intermediates. Theory may also prove to rationally guide us to novel bimetallic catalyst compositions for new study. As we master the technique of multiple metal nanoparticle synthesis, we now enter into an era of the rational design of catalysts. In principal, we can now synthesize catalysts optimized in size, shape, and composition for a 
particular reaction. With the information provided on surface reaction intermediates by SFG-VS we can make educated guesses on appropriate promoters to add to a catalyst surface. The old dream of catalysis researchers to use in-situ techniques to learn the fundamental principles of catalytic selectivity has come to fruition, and we are now in a position to begin the rational design of optimized catalysts with the goal of $100 \%$ selectivity and high activity.

\section{ACKNOWLEDGMENT}

This work was supported by the Director, Office of Energy Research, Office of Basic Energy Sciences, and Materials Sciences Division of the U.S. Department of Energy under Contract DEAC02-05CH11231. 


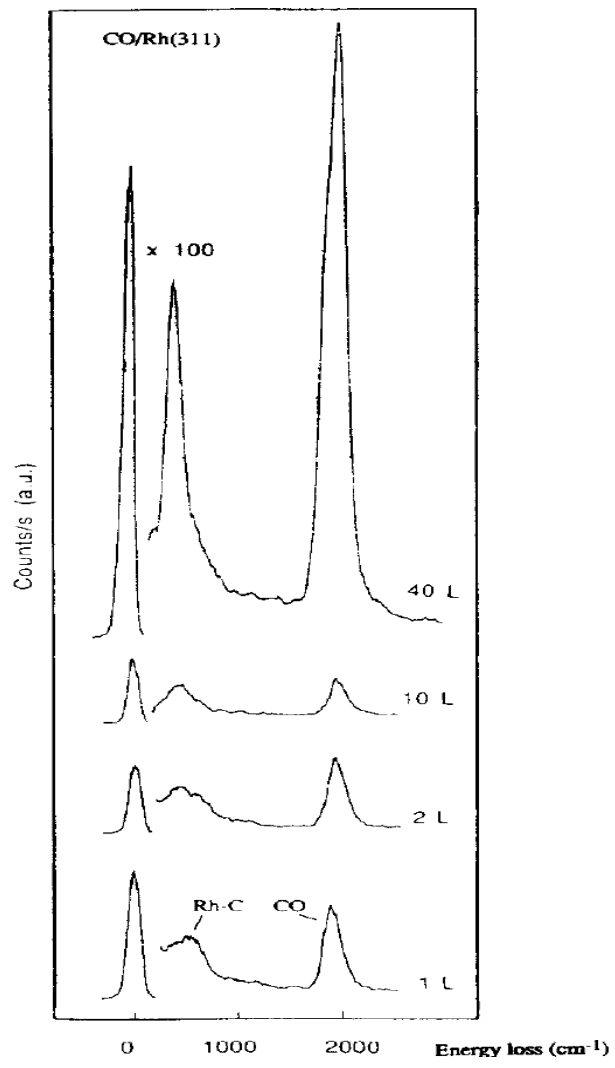

Figure 1. HREELS spectra of $\mathrm{CO}$ adsorbed onto a $\mathrm{Rh}(331)$ single crystal in the specular direction as a function of $\mathrm{CO}$ exposure. 


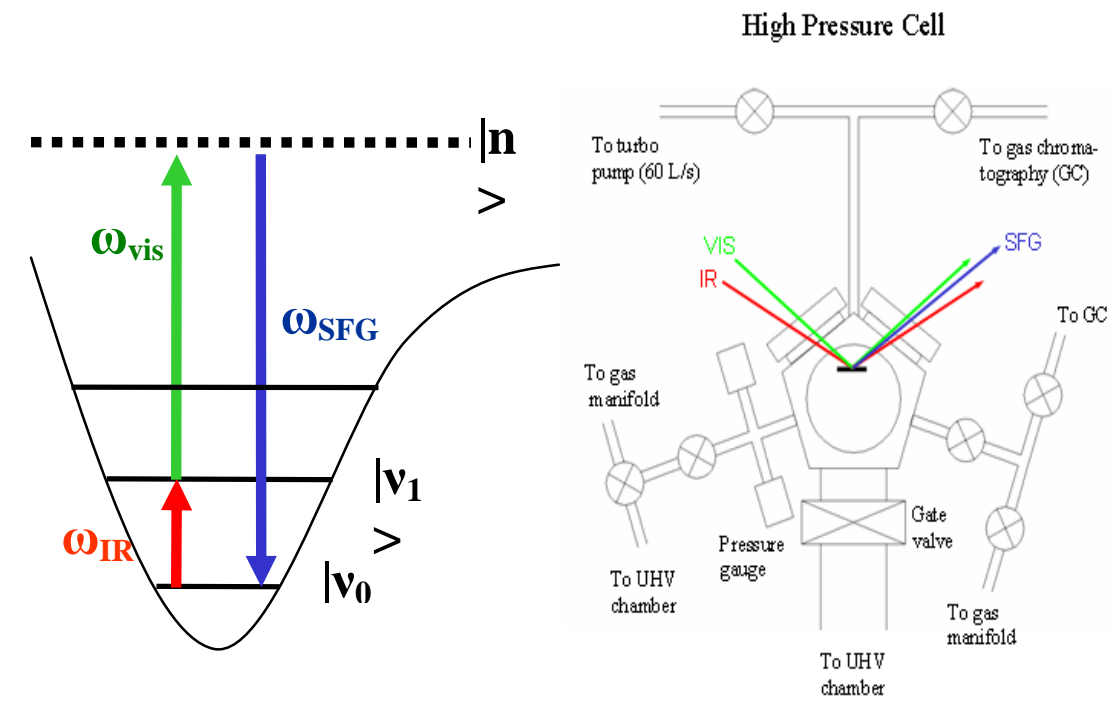

(a)

(b)

Figure 2. A) Schematic showing the SFG-VS process. The overall process will be resonantly enhanced when $\omega_{\mathrm{IR}}$ is at the same frequency as a natural vibrational mode of a molecule at the interface. B) Diagram of a typical high pressure cell for use with a SFG-VS studies of catalytic reactions at high pressure. 
4 Torr Pyrrole and 40 Torr $\mathrm{H}_{2}$ over $\operatorname{Pt}(111)$

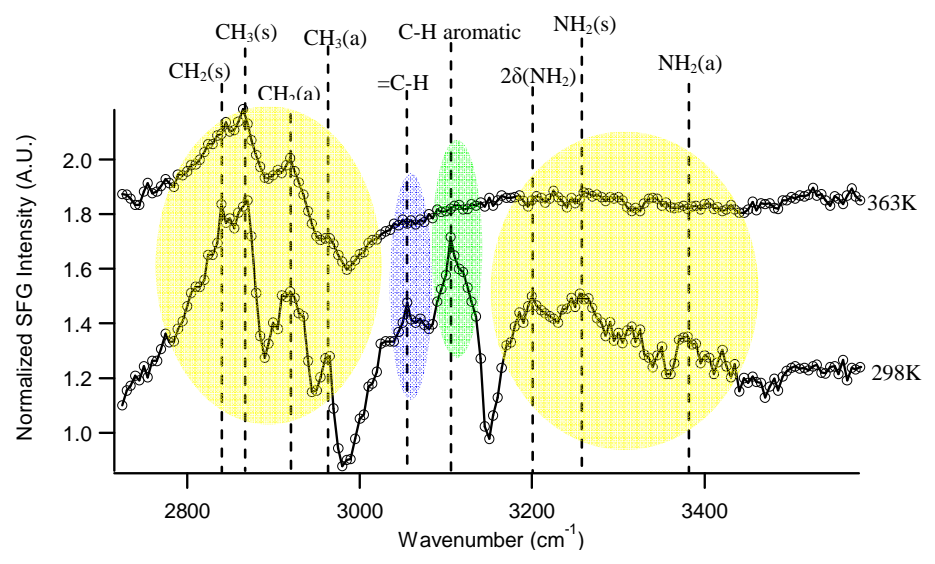

Proposed Reaction Mechanism over Pt(111)

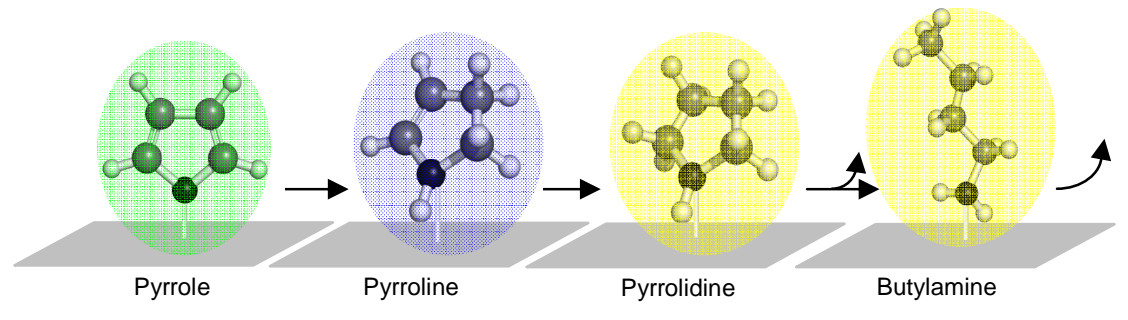

Figure 3. IR-visible SFG-VS spectra of pyrrole hydrogenation over $\operatorname{Pt}(111)$ clarifies the reaction mechanism and dominant surface species. 


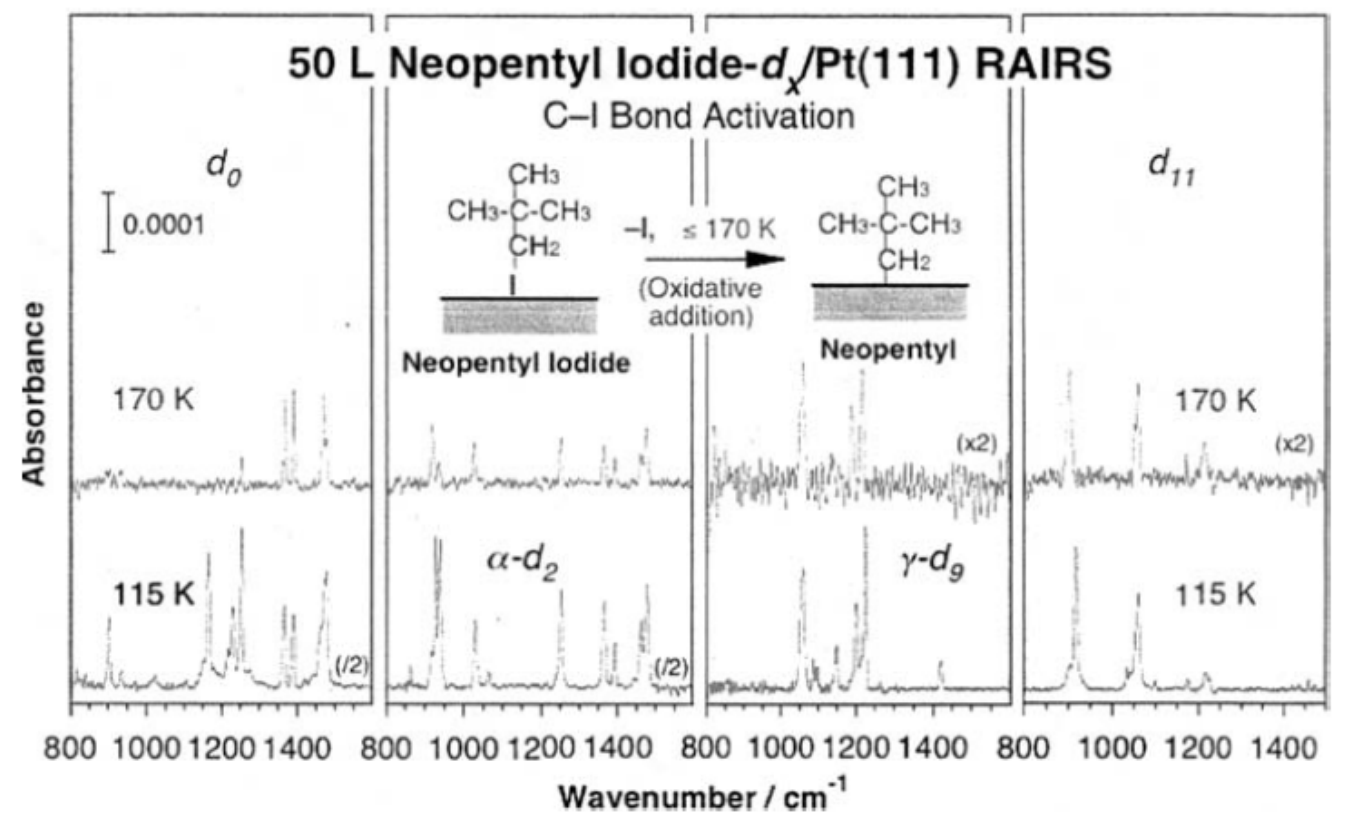

Figure 4. This figure demonstrates the preparation of a neopentyl surface intermediate taken with permission from Ref. 25. 


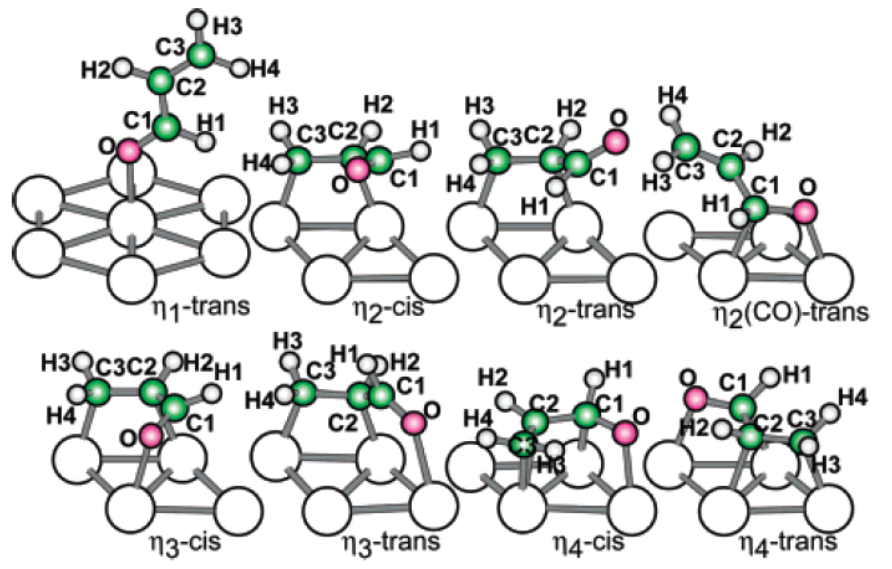

(a)

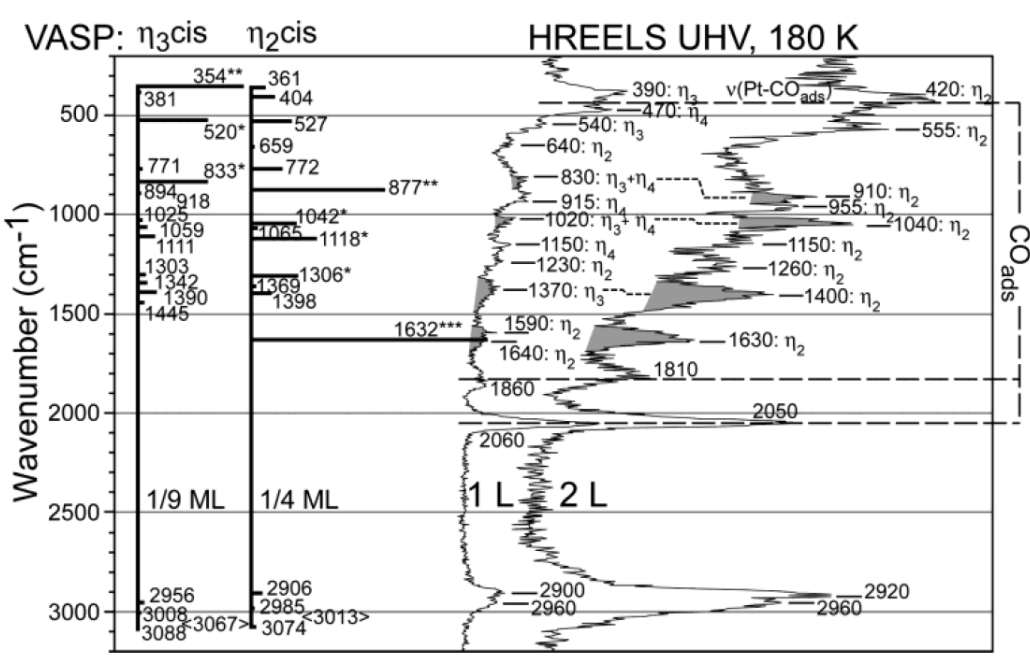

(b)

Figure 5. A) The possible adsorption structures of acrolein on $\operatorname{Pt}(111)$ energy minimized in configuration space. B) The theoretically simulated EELS spectra of two possible intermediates compared with the observed HREELS spectrum observed at a 1 Langmuir and 2 Langmuir exposure of acrolein to Pt(111). Figure taken with publisher's permission from Ref. 26. 


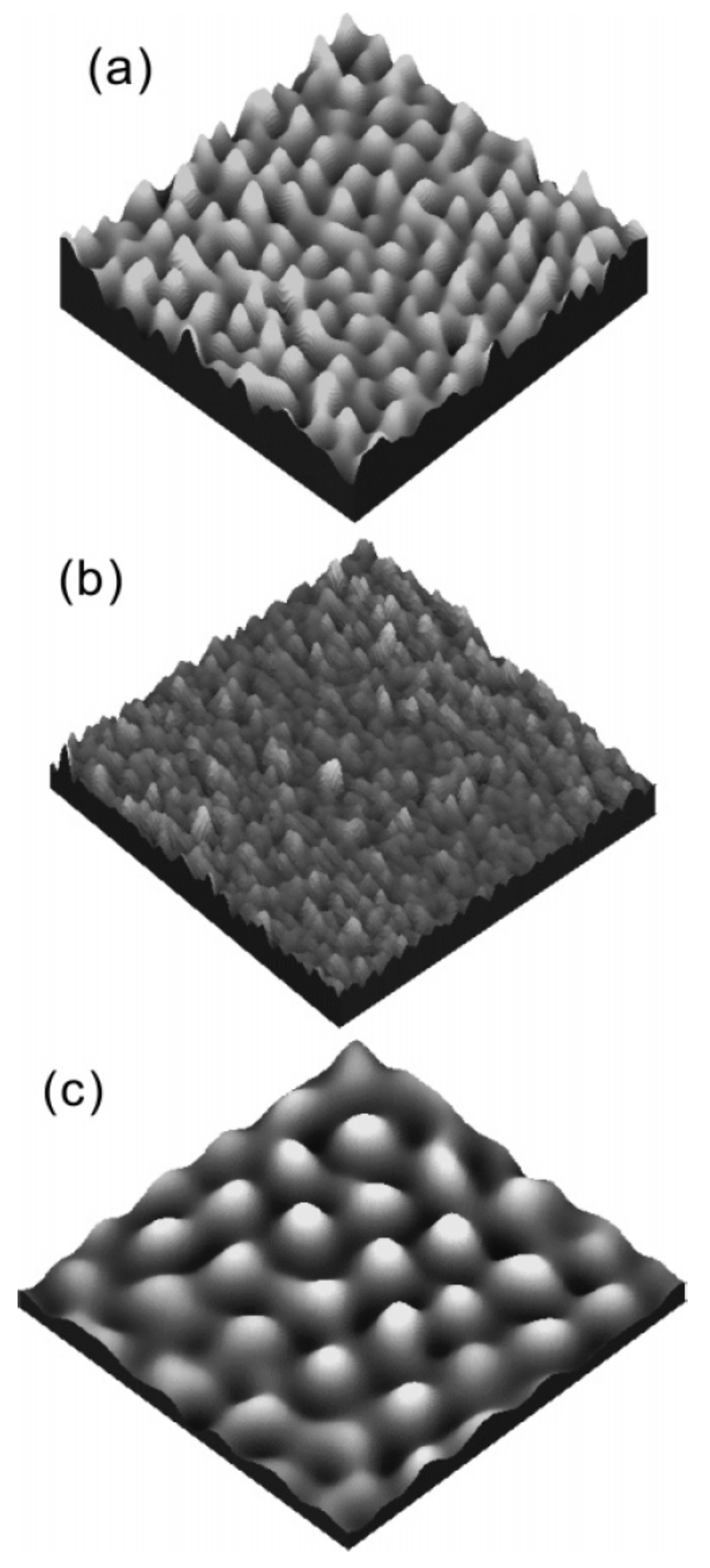

Figure 6. A) A $70 \AA$ by $70 \AA$ image of $\operatorname{Pt}(111)$ at room temperature with 20 mTorr cyclohexene and $20 \mathrm{mTorr}_{2}$, a catalytically inactive surface. B( Background pressure of hydrogen is increased to 200 mTorr making it catalytically active. C) 200 mTorr $\mathrm{H}_{2}, 20$ mTorr cyclohexene, and $5 \mathrm{mTorr}$ $\mathrm{CO}$. The $\mathrm{CO}$ has poisoned the reaction. 
a

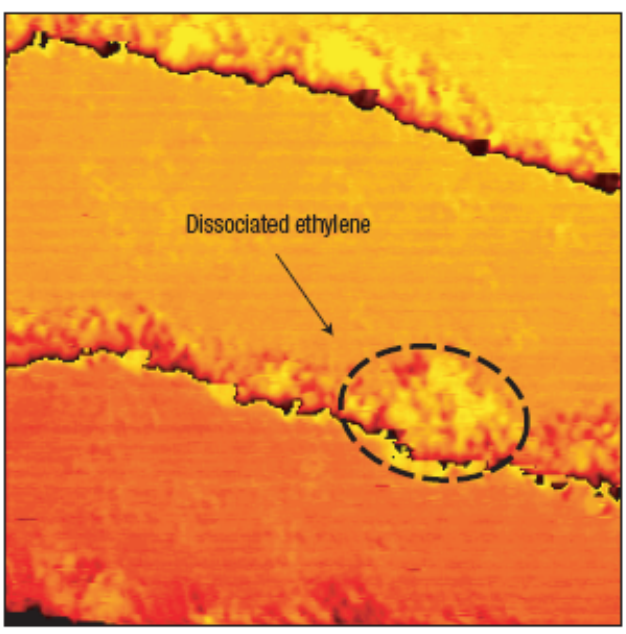

b

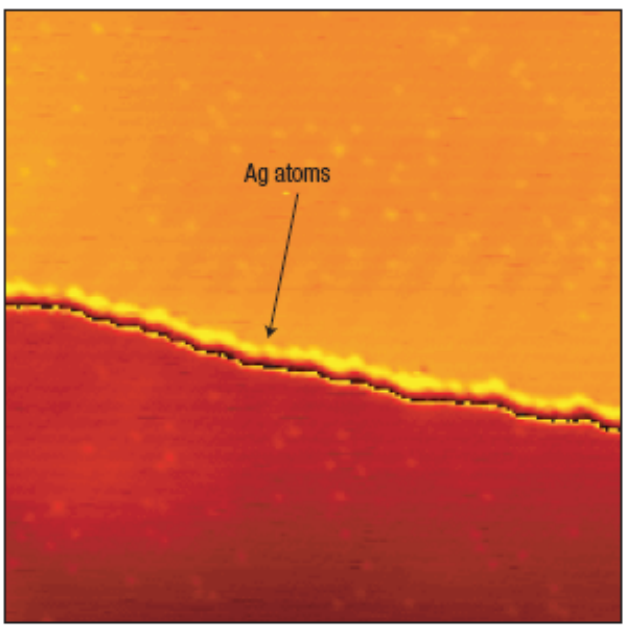

Figure 7. Ethylene decomposition over $\mathrm{Ni}(111)$. A) After exposure to $10^{-8}$ torr for $100 \mathrm{sec}$ at room temp. B) A Ag pretreated $\mathrm{Ni}(111)$ surface displays no ethylene decomposition around the step edge. Figure reproduced with publisher's permission from Ref. 41. 


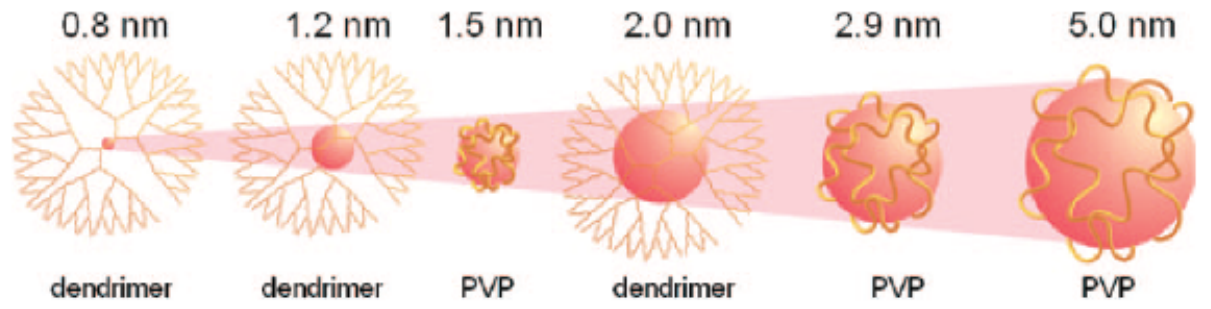

Scheme 1. Size control of Pt nanoparticles. 

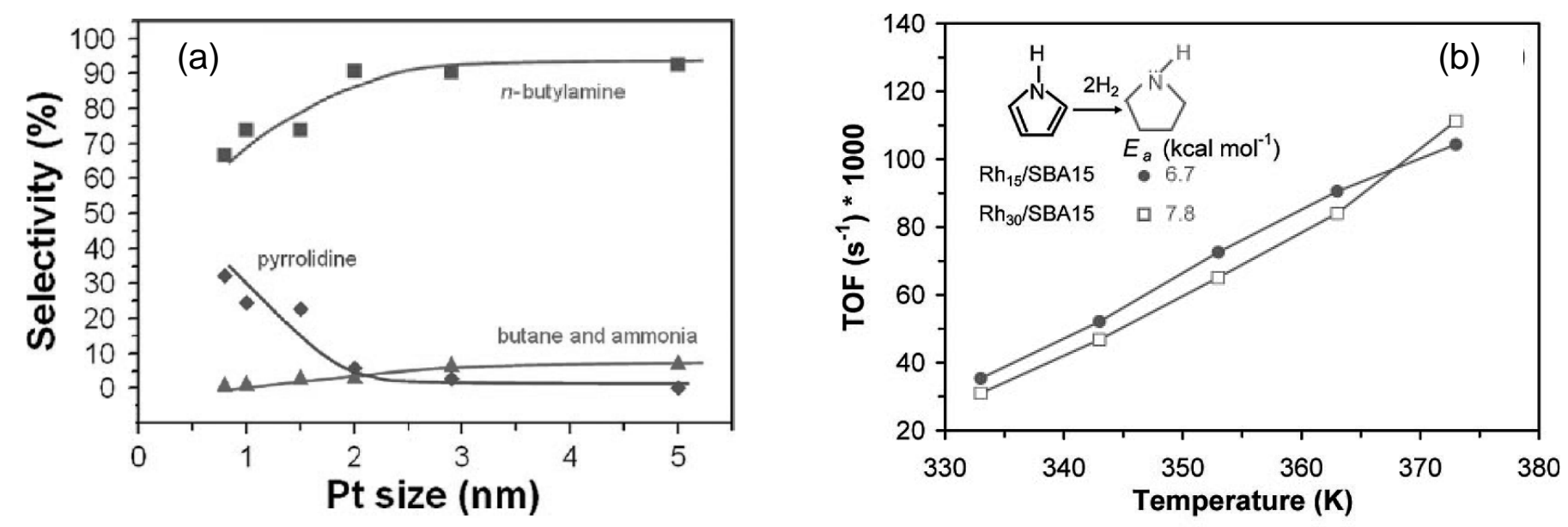

Figure 8. Particle size dependence of the selectivity for the hydrogenation of pyrrole to pyrrolidine, butylamine, and butane over $\mathrm{Pt}$ (a) and $\mathrm{Rh}$ (b) nanoparticles. Rh nanoparticles are $100 \%$ selective for the saturated ring while Pt reaction selectivity is size dependent. 

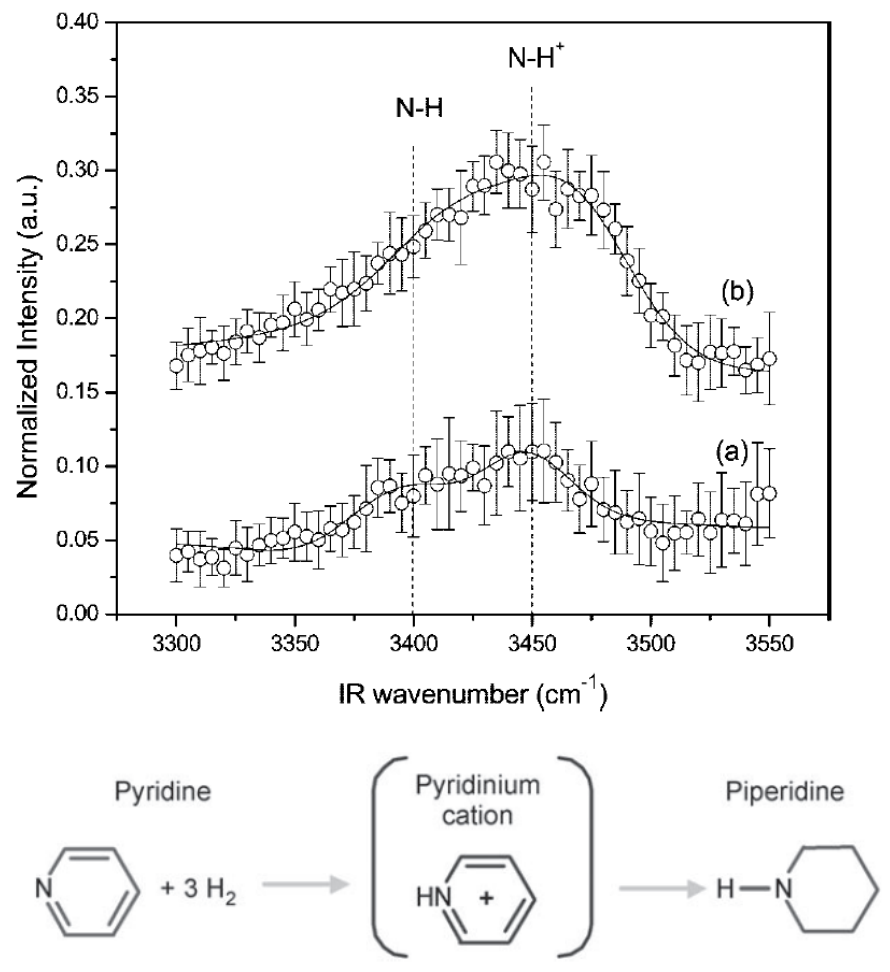

Figure 9. Pyridine hydrogenation carried out over TTAB-stabilized cubic nanoparticles revealed the pyridinium cation as the reaction intermediate. 
(1) Zaera, F.; Springer/Plenum Publishers: 2005, p 129-141.

(2) Priestnall, M. A.; Kotzeva, V. P.; Fish, D. J.; Nilsson, E. M.; Elsevier Science Bv: 2002, p 21-30.

(3) Batteas, J. D.; Gardin, D. E.; Van Hove, M. A.; Somorjai, G. A. Surface Science 1993, 297, 11-18.

(4) Wilson, J.; Guo, H.; Morales, R.; Podgornov, E.; Lee, I.; Zaera, F. Physical Chemistry Chemical Physics 2007, 9, 3830-3852.

(5) Shen, Y. R. Nature 1989, 337, 519-525.

(6) Su, X. C.; Jensen, J.; Yang, M. X.; Salmeron, M. B.; Shen, Y. R.; Somorjai, G. A.; Royal Soc Chemistry: 1996, p 263-274.

(7) Su, X. C.; Cremer, P. S.; Shen, Y. R.; Somorjai, G. A. Journal of the American Chemical Society 1997, 119, 3994-4000.

(8) McCrea, K.; Parker, J. S.; Chen, P. L.; Somorjai, G. Surface Science 2001, 494, 238-250.

(9) Cremer, P. S.; Su, X. C.; Shen, Y. R.; Somorjai, G. A. Journal of the American Chemical Society 1996, 118, 2942-2949.

(10) McCrea, K. R.; Somorjai, G. A. Journal of Molecular Catalysis a-Chemical 2000, $163,43-53$.

(11) Cremer, P. S.; Su, X. C.; Shen, Y. R.; Somorjai, G. Journal of the Chemical Society-Faraday Transactions 1996, 92, 4717-4722.

(12) Su, X. C.; Kung, K.; Lahtinen, J.; Shen, R. Y.; Somorjai, G. A. Catalysis Letters 1998, 54, 9-15.

(13) Su, X. C.; Kung, K. Y.; Lahtinen, J.; Shen, Y. R.; Somorjai, G. A. Journal of Molecular Catalysis a-Chemical 1999, 141, 9-19.

(14) Bratlie, K. M.; Montano, M. O.; Flores, L. D.; Paajanen, M.; Somorjai, G. A. Journal of the American Chemical Society 2006, 128, 12810-12816.

(15) Bratlie, K. M.; Kliewer, C. J.; Somorjai, G. A. Journal of Physical Chemistry B 2006, 110, 17925-17930.

(16) Kliewer, C. J.; Bieri, M.; Somorjai, G. A. Journal of Physical Chemistry C 2008, $112,11373-11378$.

(17) Strongin, D. R.; Somorjai, G. A. Journal of Catalysis 1988, 109, 51-60.

(18) Rupprechter, G. In Advances in Catalysis, Vol 51; Elsevier Academic Press Inc: San Diego, 2007; Vol. 51, p 133-263.

(19) Rupprechter, G.; Elsevier Science Bv: 2007, p 3-17.

(20) Wallace, W. T.; Cai, Y.; Chen, M. S.; Goodman, D. W. Journal of Physical Chemistry B 2006, 110, 6245-6249.

(21) York, R. L.; Holinga, G. J.; Guyer, D. R.; McCrea, K. R.; Ward, R. S.; Somorjai, G. A. Applied Spectroscopy 2008, 62, 937-940.

(22) Zaera, F. Applied Catalysis a-General 2002, 229, 75-91.

(23) Zaera, F. Journal of Physical Chemistry B 2002, 106, 4043-4052.

(24) Zaera, F. Catalysis Letters 2003, 91, 1-10.

(25) Janssens, T. V. W.; Zaera, F. Surface Science 2002, 501, 1-15.

(26) Loffreda, D.; Jugnet, Y.; Delbecq, F.; Bertolini, J. C.; Sautet, P. Journal of Physical Chemistry B 2004, 108, 9085-9093.

(27) Montano, M.; Salmeron, M.; Somorjai, G. A. Surface Science 2006, 600, 18091816. 
(28) Montano, M.; Tang, D. C.; Somorjai, G. A. Catalysis Letters 2006, 107, 131-141.

(29) Montano, M. O.; Salmeron, M.; Somorjai, G. A. Abstracts of Papers of the American Chemical Society 2006, 231.

(30) Cremer, P. S.; McIntyre, B. J.; Salmeron, M.; Shen, Y. R.; Somorjai, G. A. Catalysis Letters 1995, 34, 11-18.

(31) Poon, H. A.; Salmeron, M. B.; Somorjai, G. A. Abstracts of Papers of the American Chemical Society 1996, 211, 153-COLL.

(32) Somorjai, G. A. Journal of Molecular Catalysis a-Chemical 1996, 107, 39-53.

(33) Somorjai, G. A.; Su, X. C.; McCrea, K. R.; Rider, K. B. Topics in Catalysis 1999, 8, 23-34.

(34) Rider, K. B.; Hwang, K. S.; Salmeron, M.; Somorjai, G. A. Journal of the American Chemical Society 2002, 124, 5588-5593.

(35) Tang, D. C.; Hwang, K. S.; Salmeron, M.; Somorjai, G. A. Journal of Physical Chemistry B 2004, 108, 13300-13306.

(36) Somorjai, G. A.; Bratlie, K. M.; Montano, M. O.; Park, J. Y. Journal of Physical Chemistry B 2006, 110, 20014-20022.

(37) Somorjai, G. A. Journal of Physical Chemistry B 2002, 106, 9201-9213.

(38) Taylor, H. S. Proceedings of the Royal Society of London Series a-Containing Papers of a Mathematical and Physical Character 1925, 108, 105-111.

(39) Gwathmey, A. T.; Cunningham, R. E. Advances in Catalysis 1958, 10, 57-95.

(40) Somorjai, G. Introduction to Surface Science and Catalysis, 1994.

(41) Vang, R. T.; Honkala, K.; Dahl, S.; Vestergaard, E. K.; Schnadt, J.; Laegsgaard,

E.; Clausen, B. S.; Norskov, J. K.; Besenbacher, F. Nature Materials 2005, 4, 160-162.

(42) Kuhn, J. N.; Huang, W. Y.; Tsung, C. K.; Zhang, Y. W.; Somorjai, G. A. Journal of the American Chemical Society 2008, 130, 14026-+.

(43) Huang, W.; Kuhn, J. N.; Tsung, C. K.; Zhang, Y.; Habas, S. E.; Yang, P.; Somorjai, G. A. Nano Letters 2008, 8, 2027-2034.

(44) Zhang, Y. W.; Grass, M. E.; Kuhn, J. N.; Tao, F.; Habas, S. E.; Huang, W. Y.;

Yang, P. D.; Somorjai, G. A. Journal of the American Chemical Society 2008, 130, 5868-+.

(45) Koebel, M. M.; Jones, L. C.; Somorjai, G. A. Journal of Nanoparticle Research 2008, 10, 1063-1069.

(46) Lee, H.; Habas, S. E.; Kweskin, S.; Butcher, D.; Somorjai, G. A.; Yang, P. D. Angewandte Chemie-International Edition 2006, 45, 7824-7828.

(47) Vig, J. R. Journal of Vacuum Science \& Technology a-Vacuum Surfaces and Films 1985, 3, 1027-1034.

(48) Bratlie, K. M.; Komvopoulos, K.; Somorjai, G. A. Journal of Physical Chemistry C 2008, 112, 11865-11868.

(49) Tao, F.; Grass, M. E.; Zhang, Y. W.; Butcher, D. R.; Renzas, J. R.; Liu, Z.;

Chung, J. Y.; Mun, B. S.; Salmeron, M.; Somorjai, G. A. Science 2008, 322, 932-934.

(50) Zhang, Y. W.; Huang, W. Y.; Habas, S. E.; Kuhn, J. N.; Grass, M. E.; Yamada, Y.; Yang, P.; Somorjai, G. A. Journal of Physical Chemistry C 2008, 112, 12092-12095.

(51) Park, J. Y.; Zhang, Y.; Grass, M.; Zhang, T.; Somorjai, G. A. Nano Letters 2008, $8,673-677$.

(52) Studt, F.; Abild-Pedersen, F.; Bligaard, T.; Sorensen, R. Z.; Christensen, C. H.; Norskov, J. K. Science 2008, 320, 1320-1322. 
